\title{
Species concepts and malaria parasites: detecting a cryptic species of Plasmodium
}

\author{
Susan L. Perkins $\dagger$ \\ Department of Biology, University of Vermont, Burlington, VT 05405, USA
}

\begin{abstract}
Species of malaria parasite (phylum Apicomplexa: genus Plasmodium) have traditionally been described using the similarity species concept (based primarily on differences in morphological or life-history characteristics). The biological species concept (reproductive isolation) and phylogenetic species concept (based on monophyly) have not been used before in defining species of Plasmodium. Plasmodium azurophilum, described from Anolis lizards in the eastern Caribbean, is actually a two-species cryptic complex. The parasites were studied from eight islands, from Puerto Rico in the north to Grenada in the south. Morphology of the two species is very similar (differences are indistinguishable to the eye), but one infects only erythrocytes and the other only white blood cells. Molecular data for the cytochrome $b$ gene reveal that the two forms are reproductively isolated; distinct haplotypes are present on each island and are never shared between the erythrocyte-infecting and leucocyte-infecting species. Each forms a monophyletic lineage indicating that they diverged before becoming established in the anoles of the eastern Caribbean. This comparison of the similarity, biological and phylogenetic species concepts for malaria parasites reveals the limited value of using only similarity measures in defining protozoan species.
\end{abstract}

Keywords: Plasmodium; species concepts; cryptic species; malaria

\section{INTRODUCTION}

In 1842, Charles Darwin sketched out a lengthy outline of what would become the Origin of species, then set it aside for many years while he assembled the necessary background information in support of his major hypotheses. Primary to this effort was the need to clarify the concept of species (Darwin 1958). A century and a half later, this goal still presents a vexing problem in modern biology and generates substantial controversy. The number of different species concepts continues to grow; indeed Mayden (1997) catalogued 22 species concepts that are currently in use. These definitions can be grouped into three broad classes: similarity species concepts that are based on unique phenotypic features of the organisms; biological or reproductive species concepts that centre on the ability of sexually reproducing organisms to cross and produce fertile offspring; and phylogenetic or lineagebased concepts that emphasize shared evolutionary history of populations (monophyly). Ideally, descriptions of species should address each of these three main types of concepts, but in practice this is often not feasible.

The malaria parasites (phylum Apicomplexa: genus Plasmodium), present a case study in the difficulties of defining species. Until recently, Plasmodium species were described based entirely on phenotypic similarity measures including morphology of the stages infecting vertebrate blood cells, life-history traits, species of host infected or symptoms of infection (Garnham 1966). These may be unreliable characteristics, however. For example, the parasite cells seen under the microscope have been severely distorted in appearance by preservation and staining, parasites may infect a wide range of host species, and life-history traits vary substantially among infections

$\dagger$ Present address: Division of Invertebrates, American Museum of Natural History, Central Park West at 79th St, New York, NY 10024, USA (sperkins@amnh.org). for a given species of parasite (Buckling et al. 1997; Escalante et al. 1998; Eisen \& Schall 2000).

The biological species concept (BSC) defines species as 'groups of actively or potentially interbreeding populations which are reproductively isolated from other such populations' (Mayr 1963). Although it has long been popular among taxonomists of metazoan organisms, the BSC is inadequate for many parasites including Plasmodium because it uses criteria with severe limitations for asexually reproducing organisms. Although Plasmodium does have a sexually reproductive stage, almost no explicit data are available on reproductive isolation among defined species. In fact, a debate continues over the degree of clonality in these protozoan parasites (Dye 1991; Tibayrenc \& Ayala 1991; Walliker 1991; Paul et al. 1995; De Meeûs et al. 1998). Finally, the degree to which populations of parasites may overlap and potentially interbreed can largely be a function of their hosts' distribution, both past and present (KillickKendrick 1978), and not a limitation of their own reproductive compatibilities.

The advent of molecular genetic techniques such as DNA sequencing has allowed a novel insight into the definition of species. Cracraft (1983) defined species under a lineage-based perspective, termed the phylogenetic species concept, as 'the smallest diagnosable cluster of individual organisms within which there is a pattern of ancestry and descent'. The phylogenetic species concept has been used to describe numerous instances of cryptic species complexes, groups of organisms that are indistinguishable morphologically, yet show as much genetic differentiation as described species. Examples include well-studied insect systems such as Anopheles mosquitoes (White 1974; Lounibos et al. 1998), other invertebrate taxa (Sturmbauer et al. 1999), reptiles (Bruna et al. 1996) and even large birds (Baker et al. 1995). Many parasite species have also been found to comprise species complexes (e.g. Lymbery \& Thompson 1996; Hung et al. 1999). Molecular 


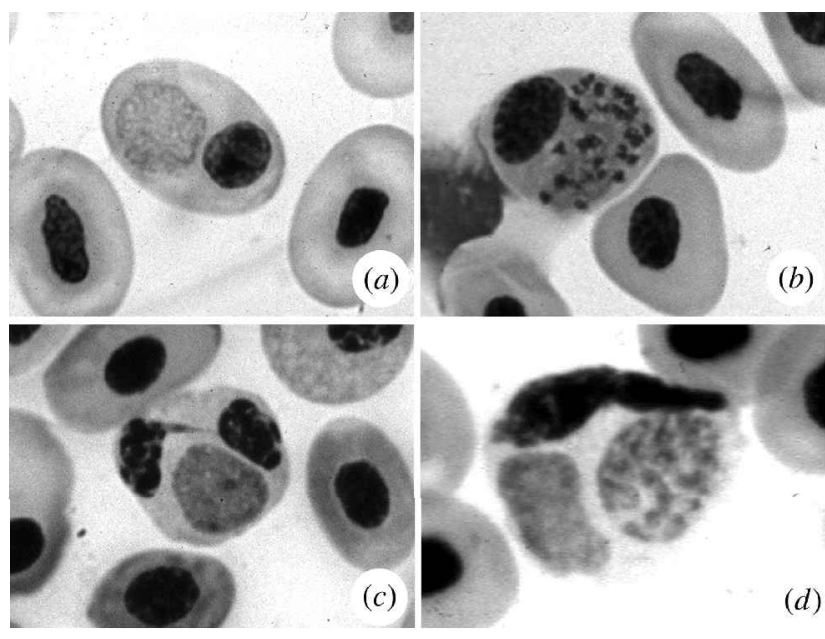

Figure 1. P. azurophilum sensu Telford (1975) in lizard blood cells. (a) Gametocyte in erythrocyte. (b) Schizont in erythrocyte. (c) Gametocyte in leucocyte. (d) Schizont (and gametocyte) in leucocyte.

data are beginning to help uncover the relationships for some species of Plasmodium (primarily those infecting mammals), but such data on intraspecific relationships are scant. Exceptions to this are the classic studies by Walliker and others (summarized in Beale \& Walliker 1988) on enzymatic differences of various rodent malaria subspecies and recent work concerning the human malaria parasite, Plasmodium vivax (Qari et al. 1993; Escalante et al. 1995). Here I present evidence for one such instance of a cryptic species of Plasmodium and support my conclusions with data relevant for defining these parasites based on similarity, biological (reproductive) and phylogenetic species concepts.

Plasmodium azurophilum is a malaria parasite of Anolis lizards in the Caribbean islands. In his species description, Telford (1975) noted that these parasites appeared to undergo both asexual replication (schizogony) and production of sexual stages or gametocytes (gamegony) in both erythrocytes and several classes of white blood cells (figure 1). This was unusual because at the time only one other Plasmodium species (Plasmodium mexicanum) had been noted to exploit cells other than erythrocytes of the vertebrate host, and then only in exceedingly heavy infections (Ayala 1970; Jordan 1970). Telford (1975) found no morphological differences for parasites in erythrocytes versus leucocytes, and data from course-of-infection studies suggested that the parasites might be switching from one host cell class to another during their life cycle. A few years later, Ayala \& Hertz (1981) reported P. azurophilum in Anolis roquet lizards from Martinique, yet noted that the forms in erythrocytes and those in leucocytes were never observed in the same lizard, nor even at the same collecting site, and they concluded that, 'were it not for Telford's thorough description, our plasmodia would unhesitatingly have been identified as two distinct species'. In a subsequent study of P. azurophilum from St Martin island, Schall (1992) observed sites and even occasionally individual lizards with both forms, yet noted that most P. azurophilum infections were exclusively either in erythrocytes or in leucocytes and proposed that the parasites must quickly cycle from one cell class to another during an infection.

Here I show using DNA sequencing of P.azurophilum sensu Telford that these parasites are in fact composed of two genetically distinct lineages - one that infects erythrocytes and one that infects white blood cells. Although the two parasites are indistinguishable morphologically under the light microscope (except for the obvious distinction of host cell class), they are reproductively isolated and have had independent evolutionary histories in the Caribbean. This appears to be the first such comparative data for any malaria parasite.

\section{MATERIAL AND METHODS}

\section{(a) Parasite sampling}

P. azurophilum is found throughout the eastern Caribbean, from Puerto Rico in the north to Grenada in the south (Staats \& Schall 1996a; this study). Infected lizards were collected from eight islands (Puerto Rico, Saba, St Kitts, Guadeloupe, Dominica, Martinique, St Vincent and Grenada) either by hand or by slip noose. A toe clip was used to obtain blood both for a thin smear and to blot filter paper for subsequent DNA extraction. All lizards were returned to their place of capture within $24 \mathrm{~h}$ as per the protocol approved by the University of Vermont Animal Care and Use Committee. Thin smears were stained with Giemsa and scanned for six or more minutes at $\times 1000$. Only those infections in which parasites were observed exclusively in one cell class were used for any further genetic work; however, mixed infections were used for morphological measurements since each cell was measured independently and classified by host cell type (erythrocyte or leucocyte).

\section{(b) Morphometric tests}

Parasites were measured from 12 natural infections from Anolis gundlachi from the El Verde Field Station, Puerto Rico. Each blood smear was scanned at $\times 1000$ and parasites were chosen for measurement if judged to be of mature morphology (largest size for both schizonts and gametocytes, largest number of merozoites for schizonts and distinct staining for male and female gametocytes). Parasite cells were measured using a drawing tube and a ZIDAS (Zeiss, Thornwood, NY, USA) image analysis system. Three size measurements were taken for each parasite: (i) parasite cell area in square micrometres; (ii) parasite maximum diameter in micrometres; and (iii) parasite minimum diameter in micrometres. Three measures of parasite cell shape were also taken: (i) parasite minimum diameter/ maximum diameter; (ii) parasite circle diameter (the area of an irregular shape transformed into an equivalent circle area); and (iii) parasite form factor $\left((4 \pi\right.$ area $) /$ perimeter $\left.^{2}\right)$.

\section{(c) DNA extraction, amplification and sequencing}

For each island, six infections were chosen for genetic analysis, three consisting of parasites only in erythrocytes and three with parasites only in white blood cells, with two exceptions. On Grenada, infections were found only in erythrocytes, and on Guadeloupe just one lizard was found to be infected with P. azurophilum in erythrocytes only and just one lizard was infected with P.azurophilum in white blood cells only. DNA was extracted from blood dried on the filter paper using sodium dodecyl sulphate lysis followed either by phenol-chloroform extraction, and ethanol precipitation and washing, or by ammonium acetate $(7.5 \mathrm{M})$ precipitation of proteins with subsequent 


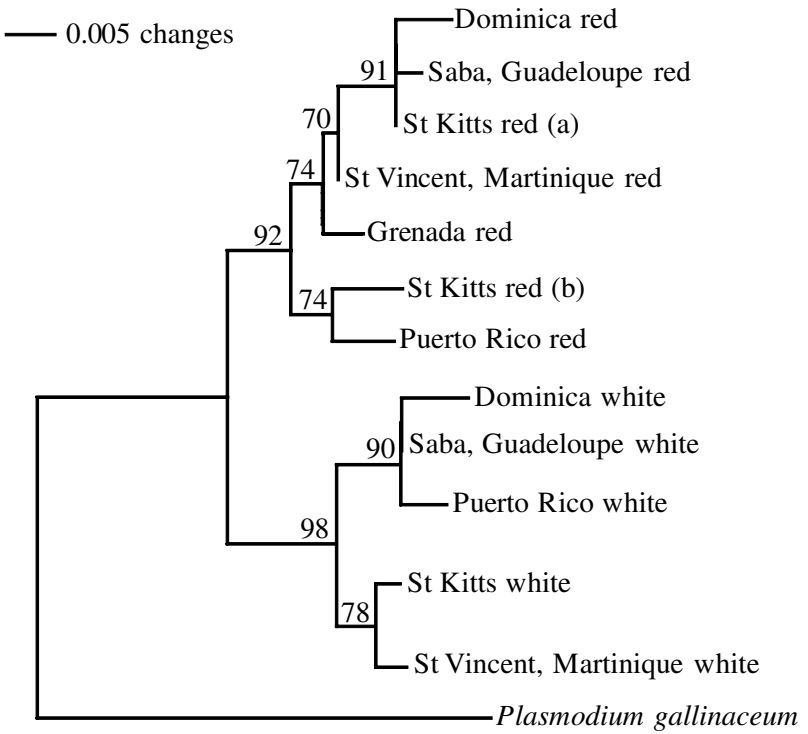

Figure 2. Neighbour-joining tree of P. azurophilum haplotypes, rooted with the avian malaria parasite, $P$. gallinaceum. Red, infection in erythrocytes; white, infection in leukocytes. Node support was determined with 1000 replicates of jackknifing with $33 \%$ deletion in each.

isopropanol precipitation and ethanol washing. A fragment of the mitochondrial cytochrome $b$ gene was amplified using primers DW1 (5'-TCAACAATGACTTTATTTGG-3') and DW3 $\left(5^{\prime}\right.$-TGCTGTATCATACCCTAAAG-3'), which produce a 673 bp product (Creasey et al. 1993). Reactions were set up in $25 \mu \mathrm{l}$ PCRs with Ready-to-Go ${ }^{\mathrm{TM}}$ PCR beads (Pharmacia Biotech, Piscataway, NJ, USA) using $1.5 \mathrm{mM} \mathrm{MgCl}_{2}, 2.5 \mu \mathrm{M}$ of each primer and ca. $250 \mathrm{ng}$ of genomic DNA. Amplification reactions were subjected to 35 cycles of $94^{\circ} \mathrm{C}$ for $1 \mathrm{~min}, 48^{\circ} \mathrm{C}$ for $1 \mathrm{~min}$, and $72{ }^{\circ} \mathrm{C}$ for $1 \mathrm{~min}$ and then $72{ }^{\circ} \mathrm{C}$ for $5 \mathrm{~min}$. For weak infections, a nested PCR design was employed using outer primers DW2 (5'-TAATGCCTAGACGTATTCCTGATTATC CAG-3', self-designed) and DW4 (5'-TGTTTGCTTGGGAGC TGTAATCATAATGTG-3' = AL1356; Escalante et al. 1998) and subjected to 35 cycles of $94^{\circ} \mathrm{C}$ for $1 \mathrm{~min}, 60^{\circ} \mathrm{C}$ for $1 \mathrm{~min}$ and $72^{\circ} \mathrm{C}$ for $1 \mathrm{~min}$ with a final polymerization step of $5 \mathrm{~min}$ at $72^{\circ} \mathrm{C}$. A 0.51 aliquot of this product was used as a template for a nested reaction with primers DW1 and DW3 under the same conditions as above. PCR products were purified with Nanosep 100K columns (Pall Gelman, Ann Arbor, MI, USA), subjected to cycle sequencing using both forward and reverse primers (DW1 and DW3) and run on an ABI (Applied Biosystems, Foster City, CA, USA) Prism automated sequencer (Vermont Cancer Center, University of Vermont).

\section{(d) Phylogenetic analysis}

A total of $554 \mathrm{bp}$ of the cytochrome $b$ gene were used for this analysis $\left(5^{\prime}\right.$ nucleotides were removed so that the sequences could be aligned with the published sequence for Plasmodium gallinaceum, an avian malaria parasite that was used as the outgroup, GenBank accession numbers AF069612). All phylogenetic analyses were done using PAUP* 4.0b4 (Swofford 1999). Three methods of tree construction were employed: neighbour joining, unweighted maximum parsimony, and maximum likelihood. Neighbour-joining trees were calculated using TamuraNei distances. These were appropriate due to the high A-T bias (average $=3.5 \%)$ for these sequences (Tamura \& Nei 1984). For maximum-likelihood analyses, a set of 40 evolutionary models was tested on the neighbour-joining tree obtained from an initial search using the program ModelTest (Posada \& Crandall 1998). The simplest, best model (via log-likelihood ratio tests) was then chosen for the maximum-likelihood algorithm. This model consisted of a six-step (general time reversible) matrix, proportion of invariable sites $=0$ and gamma shape parameter of 0.0102. The Kishino-Hasegawa test $(\mathrm{K}-\mathrm{H}$ test; Kishino \& Hasegawa 1989) was used as a statistical test of alternative resolutions of the phylogeny. For this test, two competing hypotheses were compared: (i) P.azurophilum in erythrocytes and P.azurophilum in white blood cells each form monophyletic groups; versus (ii) the erythrocytic forms and white blood cellinfecting forms from each island are each other's closest relatives.

\section{RESULTS}

\section{(a) Morphometric analyses}

A discriminate analysis was used to determine the use of morphological measurements in differentiating P. azurophilum cells in erythrocytes versus leucocytes.

Discriminate scores successfully classified 46 out of 64 $(72 \%)$ of gametocytes (likelihood ratio test, $p=0.0005)$, but failed to distinguish schizonts based on cell type exploited (50 out of 82, 61\% successfully classified, $p>0.05)$. When only measurements related to parasite size (parasite area, maximum diameter, minimum diameter) were used, the discriminate analysis again successfully classified 41 out of $64(64 \%)$ of the cells $(p=0.031)$ for gametocytes, but was not reliable for schizonts (45 out of 82 or $55 \%, p<0.05$ ). Using the shape parameters alone did not allow discrimination of either gametocytes $(52 \%, p>0.05)$ or schizonts $(57 \%$, $p>0.05)$. Although the composite size measure was successful in distinguishing gametocytes in the two host cell classes, each measure alone did not differ for parasites in host erythrocytes versus leucocytes ( $U$-tests, $p>0.05)$.

\section{(b) Phylogenetic analyses}

A total of 12 haplotypes of this fragment of the cytochrome $b$ gene were identified, ranging from 0.18 to $3.1 \%$ in sequence divergence. All samples sequenced for a given cell class were identical on each island with the exception of St Kitts where two haplotypes of P. azurophilum in erythrocytes were observed. Several islands shared haplotypes: Saba and Guadeloupe for P. azurophilum in erythrocytes, Martinique and St Vincent in erythrocytes, Saba and Guadeloupe in leucocytes, and Martinique and St Vincent in leucocytes. These were pooled for all phylogenetic analyses. The alignment of each of these haplotypes showed four fixed differences between the parasites in erythrocytes and those infecting leucocytes and no haplotypes were shared between infections in red and white blood cells. It is possible that this assortment of haplotypes was simply a result of random sampling. However, the maximum probability of this occurring may be calculated as follows. If we assume that the two haplotypes occur in a 1:1 ratio (the probability would be lower if other ratios occur), then for each island with three infections in each cell class sequenced, the probability would be $(1 \times 0.5 \times 0.5)$ $\times(0.5 \times 0.5 \times 0.5)=0.03$, and the total probability would 


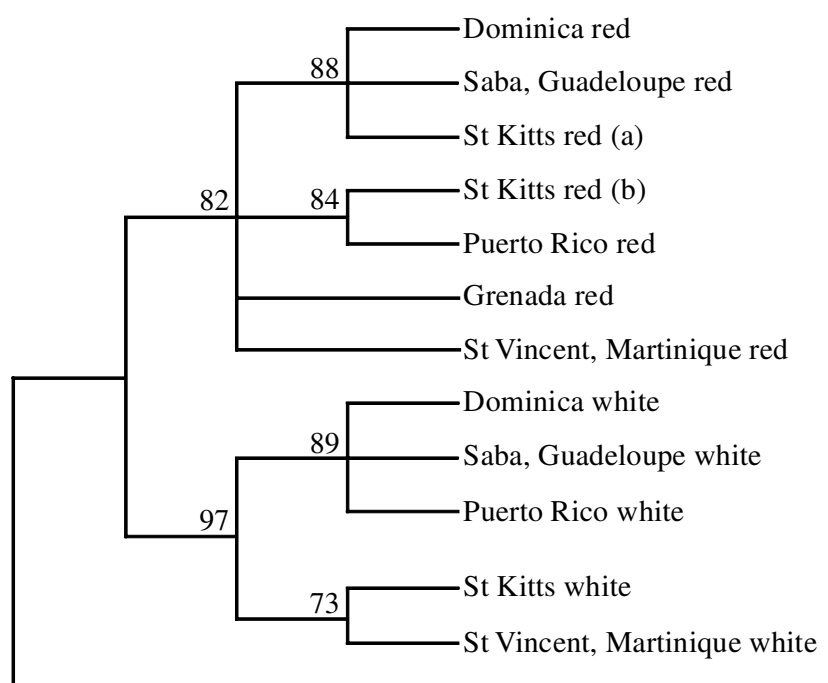

Plasmodium gallinaceum

Figure 3. Strict consensus of four equally parsimonious trees, rooted with the avian malaria parasite, P. gallinaceum. Red, infection in erythrocytes; white, infection in leucocytes. The consistency index is 0.893 and the retention index is 0.878 . The length of the tree is 56 steps. Node support was determined with 1000 replicates of jackknifing with $33 \%$ deletion of characteristics in each.

be the product over all islands. Thus, the probability of apparent division of haplotypes by cell class occurring by chance during the sampling was $5.7 \times 10^{-8}$.

All three methods of tree construction revealed two distinct monophyletic groups of parasites: one infecting erythrocytes and one infecting white blood cells (figures 2-4), each with strong nodal support for this division. The maximum-parsimony analysis produced four trees; a strict consensus is shown in figure 3. The maximumlikelihood analysis yielded the tree seen in figure 4 . The $\mathrm{K}-\mathrm{H}$ test significantly rejected the hypothesis that the erythrocytic forms and forms infecting white blood cells from each island are each other's closest relatives in favour of the monophyletic grouping of P.azurophilum in erythrocytes and P.azurophilum in leucocytes (parsimony: 56 steps versus 120 steps, $p<0.0003$; maximum likelihood: -1044 versus $-1260, p<0.0001$ ).

\section{DISCUSSION}

For the past 120 years, parasitologists have described taxa of malaria parasites using only the similarity species concept (indeed, this is the standard method for taxonomists working with any group of protozoan parasites). Morphological traits such as the number of merozoites per schizont (daughter cells produced by each asexually dividing cell), shape of the gametocytes, presence or absence of pigment, kind of host cell infected, species of host infected and symptomatology of infected hosts, have been used both to describe species and as characteristics in higher systematic treatments (Garnham 1966). These traits are likely to be unreliable for several reasons. Morphological characteristics determined from fixed, stained blood smears may be altered depending on researchers' techniques and result in significant differences in the appearance of the parasites under the light

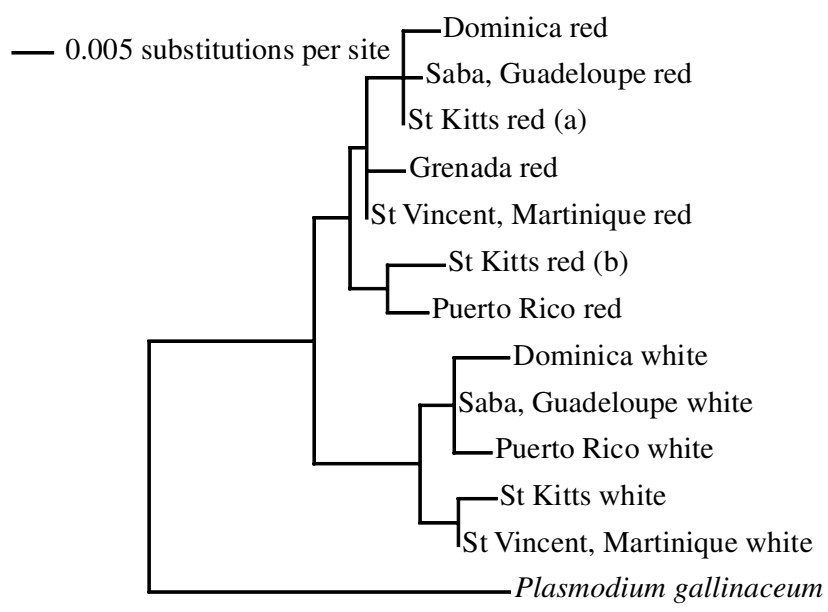

Figure 4. Maximum-likelihood phylogeny of P. azurophilum haplotypes constructed with a general time reversible (six-parameter) model of substitution, proportion of invariable sites $=0$ and a gamma shape parameter of 0.0102 , rooted with the avian malaria parasite, $P$. gallinaceum. Red, infection in erythrocytes; white, infection in leucocytes. The log-likelihood score of the phylogeny is - 1044.15.

microscope. The morphology and life history of parasites may also vary according to the host species in which they find themselves (Manwell 1943; Jordan 1975). Finally, a phylogenetic study of Plasmodium and other genera of malaria parasites (S. L. Perkins and J. J. Schall, unpublished data) has shown that both life-history traits and host species infected are uninformative for revealing evolutionary relationships among taxa. The practice of using similarity-based species concepts has been very useful, but conceivably has led to the splitting of true species based on inter-infection variation as well as the lumping of taxa that show morphological similarities.

Qari et al. (1993) used sequence data to show that the human malaria parasite $P$. vivax consists of two forms each with unique circumsporozoite genes, although morphologically the two forms are very similar. One of these forms, called P.vivax-like, is most likely Plasmodium simiovale, a parasite of New World monkeys. Escalante et al. (1995), using the same gene, found that two other human malaria parasites are genetically indistinguishable from those of non-human primates, and suggested that either host-switching has occurred within the last 15000 years or even that monkeys may be serving as reservoir hosts for the human parasites in current times. Thus, Qari et al. (1993) and Escalante et al. (1995) have used molecular data to extend the usefulness of the similarity species concept in defining species of Plasmodium; based on degree of genetic similarity or distinctiveness, they have challenged the current taxonomic labels on these malaria parasites. Here I have shown that P. azurophilum, a malaria parasite of Caribbean island lizards, is actually two species. For the first time, all three basic types of species concepts can be applied and compared in the identification of a cryptic species of Plasmodium.

\section{(a) Similarity species concept}

Under the light microscope, P. azurophilum in erythrocytes and leucocytes are very similar in morphology. Measurements revealed that the shape of the cells does 
not differ significantly, but there are slight differences in size. This is imperceptible to the eye, however. The only phenotypic trait that clearly separates them is the type of host blood cell infected. Sequence data for the cytochrome $b$ gene reveal that the two forms of $P$. azurophilum differ by as much as $3.1 \%$. This is comparable to other, recognized species of Plasmodium. For example, the same region of this gene from two species of bird malaria, P. gallinaceum (Asiatic origin) and Plasmodium elongatum (North America) differ by 3.4\% (Escalante et al. 1998).

\section{(b) Biological species concept}

The two forms of P.azurophilum are almost certainly reproductively isolated. Sampling on multiple islands revealed that haplotypes are never shared by the parasites in red and white blood cells; the probability of this being a spurious chance result is vanishingly small. Phylogenetic analysis (S. L. Perkins and J. J. Schall, unpublished data) reveals that P.azurophilum in white blood cells occurs within a clade of typical Plasmodium infecting erythrocytes, so exploitation of white blood cells must be the derived condition. How did this occur, and what prevents the two forms from hybridizing? The two forms often occur in the same population of lizards and sometimes within the same individual host, offering an opportunity for hybridization to occur in the mid-gut of the insect vector. The timing of exflagellation could differ for parasites in erythrocytes versus white blood cells (due to the relative ease of emergence from each class of cell based on membrane strength). Mating between male and female gametes in the vector's mid-gut occurs very quickly in Plasmodium, within just a few minutes after ingestion (Carter \& Nijhout 1977). Thus, once a genotype of erythrocyte-infecting P.azurophilum switched into white blood cells it could have been reproductively isolated from its ancestor form simply by the timing of mating events in the vector.

Hybridization between species of malaria parasite, natural or experimentally induced, has not been reported, although there are numerous examples of more than one species of Plasmodium infecting a given host; for example, two to four species of Plasmodium often co-occur in humans (Richie 1988; Purnomo et al. 1999), as do lizard malaria parasites in several populations (Schall \& Bromwich 1994; Staats \& Schall 1996b). Walliker (1983) found two or more species of rodent malaria commonly infecting the same animal in the Central African Republic, yet based on enzymatic differentiation he saw no indication that the species were hybridizing. Thus, whatever the mechanism keeping the two forms of P.azurophilum separate, the finding that two similar species of Plasmodium can coexist in the same host population, and be reproductively isolated, is not extraordinary.

\section{(c) Phylogenetic species concept}

The molecular phylogeny presented here indicates that P. azurophilum is composed of two evolutionary lineages and these lineages colonized the islands of the Lesser Antilles independently. If a single species of parasite colonized the islands, then later diverged into the two forms, then on each island P.azurophilum in erythrocytes and leucocytes would be each other's closest relatives. This is not the case. In fact, the parasites in erythrocytes from all islands form one clade, as do those parasites found in leucocytes. The greatest pairwise difference observed in this data set was between the two forms on Dominica, an island in the middle of the archipelago. Thus, there could not have been multiple evolutionary origins on each island of P. azurophilum in white blood cells from the ancestral line in erythrocytes.

Over 60 years ago, Manwell (1936) was prescient in stating, 'It seems to me that we should conceive of a species of malaria in genetic terms, as we do most other living things.' Now, armed with the tools of molecular genetics, it seems that we are in a position to do so. By continuing to sample previously described species of malaria parasites throughout their ranges and collecting DNA sequence data from them, no doubt some Plasmodium species will be found to actually comprise cryptic species complexes. This information will be invaluable, both for understanding the population genetic structure of Plasmodium as it relates to such things as drug resistance and virulence and for unravelling the broad evolutionary history of this very diverse group of parasites.

I thank the following people for help in the field and the laboratory: Cathy Bliss, Sarah Osgood, Anja Pearson, Jos. J. Schall, Brett Schneider, Tom Smith, Jason Wolf and especially Andrew Wargo for his help with parasite measurements. Bill Kilpatrick, Sarah Osgood, Jos. J. Schall and Mark Siddall all gave helpful comments on the manuscript. The research was funded by grants from the US National Science Foundation (NSF), the National Geographic Society and the NSF-Vermont EPSCoR program to J. J. Schall, and a Graduate Training Grant from NSF to S.L.P.

\section{REFERENCES}

Ayala, S. C. 1970 Lizard malaria in California: description of a strain of Plasmodium mexicanum and biogeography of lizard malaria in western North America. 7. Parasitol. 56, 417-425.

Ayala, S. C. \& Hertz, P. E. 1981 Malaria infection in Anolis lizards on Martinique, Lesser Antilles. Rev. Inst. Med. Trop. São Paulo 23, 12-17.

Baker, A. J., Daugherty, C. H., Colbourne, R. \& McLennan, J. L. 1995 Flightless brown kiwis of New Zealand possess extremely subdivided population structure and cryptic species like small mammals. Proc. Natl Acad. Sci. USA 92, 8254-8258.

Beale, G. H. \& Walliker, D. 1988 Biological characterization of malaria parasites. In Malaria: principles and practice of malariology (ed. W. H. Wernsdorfer \& I. McGregor), pp. 395-409. Edinburgh, UK: Churchill Livingstone.

Bruna, E. M., Fisher, R. N. \& Case, T. J. 1996 Morphological and genetic evolution appear decoupled in Pacific skinks (Squamata: Scinidae: Emoia). Proc. R. Soc. Lond. B 263, 681688.

Buckling, A. G., Taylor, L. H., Carlton, J. M. \& Read, A. F. 1997 Adaptive changes in Plasmodium transmission strategies following chloroquine chemotherapy. Proc. R. Soc. Lond. B 264, 553-559.

Carter, R. \& Nijhout, M. M. 1977 Control of gamete formation (exflagellation) in malaria parasites. Science 195, 407-409.

Cracraft, J. 1983 Species concepts and speciation analysis. Curr. Ornithol. 1, 159-187.

Creasey, A. M., Ranford-Cartwright, L. C., Moore, D. J., Williamson, D. H., Wilson, R. J. M., Walliker, D. \& Carter, R. 1993 Uniparental inheritance of the mitochondrial gene cytochrome $b$ in Plasmodium falciparum. Curr. Genet. 23, 360-364.

Darwin, C. 1958 The autobiography of Charles Darwin and selected letters (ed. F. Darwin). New York: Dover Publications. 
De Meeûs, T. Michalakis, Y. \& Renaud, F. 1998 Santa Rosa revisited: or why are there so many kinds of parasites in the 'Garden of Earthly Delights'? Parasitol. Today 14, 10-13.

Dye, C. 1991 Population genetics of nonclonal, nonrandomly mating malaria parasites. Parasitol. Today 7, 236-240.

Eisen, R. J. \& Schall, J. J. 2000 Life history of a malaria parasite (Plasmodium mexicanum): independent traits and basis for variation. Proc. R. Soc. Lond. B 267, 793-799.

Escalante, A. A., Barrio, E. \& Ayala, F. J. 1995 Evolutionary origin of human and primate malarias: evidence from the circumsporozoite protein gene. Mol. Biol. Evol. 12, 616-626.

Escalante, A. A., Freeland, D. E., Collins, W. E. \& Lal, A. A. 1998 The evolution of primate malaria parasites based on the gene encoding cytochrome $b$ from the linear mitochondrial genome. Proc. Natl Acad. Sci. USA 95, 8124-8129.

Garnham, P. C. C. 1966 Malaria parasites and other haemosporidia. Oxford, UK: Blackwell

Hung, G. C., Chilton, N. B., Beveridge, I., Zhu, X. Q., Lichtenfels, J. R. \& Gasser, R. B. 1999 Molecular evidence for cryptic species within Cylicostephanus minutus (Nematoda: Strongylidae). Int. 7. Parasitol. 29, 285-291.

Jordan, H. B. 1970 The occurrence and development of Plasmodium mexicanum in the western fence lizard Sceloporus occidentalis. 7. Protozool. 17, 86-89.

Jordan, H. B. 1975 The effect of host constitution on the development of P. floridense. F. Protozool. 22, 241-244.

Killick-Kendrick, R. 1978 Taxonomy, zoogeography and evolution. In Rodent malaria (ed. R. Killick-Kendrick \& W. Peters), pp. 1-52. London: Academic Press.

Kishino, H. \& Hasegawa, M. 1989 Evaluation of the maximum likelihood estimate of the evolutionary tree topologies from DNA sequence data, and the branching order of Hominoidea. 7. Mol. Evol. 29, 170-179.

Lounibos, L. P., Wilkerson, R. G., Conn, J. E., Hribar, L. J., Fritz, G. N. \& Danoff-Burg, J. A. 1998 Morphological, molecular, and chromosomal discrimination of cryptic Anopheles (Nyssorhynchus) (Diptera: Culicidae) from South America. 7. Med. Entomol. 35, 830-838.

Lymbery, A. J. \& Thompson, R. C. A. 1996 Species of Echinococcus: pattern and process. Parasitol. Today 12, 486-491.

Manwell, R. D. 1936 The problem of species, with special reference to the malaria parasites. Annls Trop. Med. 30, 435-439.

Manwell, R. D. 1943 Malaria infections by four species of Plasmodium in the duck and chicken and resulting parasite modifications. Am. 7. Hyg. 38, 211-223.

Mayden, R. L. 1997 A hierarchy of species concepts: the denouement in the saga of the species problem. In Species: the units of biodiversity (ed. M. F. Claridge, H. A. Dawah \& M. R. Wilson), pp. 381-424. London: Chapman \& Hall.
Mayr, E. 1963 Animal species and evolution. Oxford University Press.

Paul, R. E. L., Packer, M. J., Walmsley, M., Lagog, M., Ranford-Cartwright, L. C., Paru, R. \& Day, K. P. 1995 Mating patterns in malaria parasite populations of Papua New Guinea. Science 269, 1709-1711.

Posada, D. \& Crandall, K. A. 1998 Model test: testing the model of DNA substitution. Bioinformatics 14, 817-818.

Purnomo, A. S., Solihin, A., Gomez-Saladin, E. \& Bangs, M. J. 1999 Rare quadruple malaria infection in Irian Jaya Indonesia. 7. Parasitol. 85, 574-579.

Qari, S. H., Shi, Y. P., Goldman, I. F., Udhayakumar, V., Alpers, M. P., Collins, W. E. \& Lal, A. A. 1993 Identification of Plasmodium vivax-like human malaria parasite. Lancet 341, 780-783.

Richie, T. L. 1988 Interactions between malaria parasites infecting the same vertebrate host. Parasitology 96, 607-639.

Schall, J. J. 1992 Parasite-mediated competition in Anolis lizards. Oecologia 92, 58-64.

Schall, J. J. \& Bromwich, C. R. 1994 Interspecific interactions tested: two species of malaria parasite in a West African lizard. Oecologia 97, 326-332.

Staats, C. M. \& Schall, J. J. $1996 a$ Malarial parasites (Plasmodium) of Anolis lizards: biogeography in the Lesser Antilles. Biotropica 28, 388-393.

Staats, C. M. \& Schall, J. J. 1996b Distribution and abundance of two malarial parasites of the endemic Anolis lizard of Saba island, Netherlands Antilles. F. Parasitol. 82, 409-413.

Sturmbauer, G., Opadiya, G. B., Niederstatter, H., Riedmann, A. \& Dallinger, R. 1999 Mitochondrial DNA reveals cryptic oligochaete species differing in cadmium resistance. Mol. Biol. Evol. 16, 967-974.

Swofford, D. L. 1999 PAUP*. Phylogenetic analysis using parsimony ( ${ }^{*}$ and other methods), v. 4. Sunderland, MA: Sinauer Associates.

Tamura, F. \& Nei, M. 1984 Estimation of evolutionary distance between nucleotide sequences. Mol. Biol. Evol. 1, 269-285.

Telford Jr, S. R. 1975 Saurian malaria in the Caribbean: Plasmodium azurophilum sp. Nov., a malarial parasite with schizogony and gametogony in both red and white blood cells. Int. 7. Parasitol. 5, 383-394.

Tibayrenc, M. \& Ayala, F. J. 1991 Towards a population genetics of microorganisms: the clonal theory of parasitic protozoa. Parasitol. Today 7, 228-232.

Walliker, D. 1983 The genetic basis of diversity in malaria parasites. Adv. Parasitol. 22, 217-259.

Walliker, D. 1991 Malaria parasites: randomly interbreeding or 'clonal' populations? Parasitol. Today 7, 232-235.

White, G. B. 1974 Anopheles gambiae complex and disease transmission in Africa. Trans. R. Soc. Trop. Med. Hyg. 68, 278-301. 